CONCISE REPORT

\title{
Autoantibodies against phosphatidylserine-prothrombin complex in patients with systemic sclerosis
}

\author{
M Hasegawa, S Sato, K Yanaba, K Komura, M Yamazaki, K Takehara
}

Ann Rheum Dis 2004;63:1514-1517. doi: 10.1136/ard.2003.016659

\begin{abstract}
Objective: To determine the frequency and clinical association of antiphosphatidylserine-prothrombin complex (PS/PT) antibody (Ab) in systemic sclerosis (SSc).

Methods: Anti-PS/PT lgG Ab was examined by enzyme linked immunosorbent assay (ELISA) in 112 patients with SSc. Thirty three healthy volunteers and 30 patients with systemic lupus erythematosus (SLE) were also investigated as controls.

Results: Anti-PS/PT Ab was detected in 18/112 (16\%) patients with SSc and $10 / 30(33 \%)$ patients with SLE, whereas it was not detected in any normal controls. Anti-PS/ PT Ab was more frequently detected in patients with SSc with peripheral ischaemia and lung disease (pulmonary fibrosis and pulmonary hypertension) than in patients with SSc without the Ab. However, anti-PS/PT Ab was not associated with the severity of skin sclerosis. Importantly, two patients were negative for both lupus anticoagulant and $\mathrm{Ab}$ against cardiolipin $\beta_{2}$-glycoprotein I complex among six anti-PS/PT $\mathrm{Ab}$ positive patients with $\mathrm{SSc}$ and a thromboembolic episode. Conclusions: Anti-PS/PT Ab is associated with thromboembolism, peripheral ischaemia, and lung involvement in some patients with SSc. Examination of this Ab may be useful to recognise the risk of thromboembolism in patients with SSc.
\end{abstract}

S ystemic sclerosis (SSc) is an autoimmune disease that targets the vasculature in the skin and internal organs, such as lung, heart, and kidney, leading ultimately to fibrosis. In its later stage, SSc can be associated with vascular occlusion, thromboembolism, and peripheral ischaemia. Antiphospholipid antibodies (Abs) including lupus anticoagulant (LA) and Ab against cardiolipin $\beta_{2}$-glycoprotein I complex (anti-CL/ $\beta_{2}$ GPI $\mathrm{Ab}$ ) are thought to be involved in thrombosis in patients with antiphospholipid syndrome (APS). ${ }^{1}$ Prothrombin appears to be another antigenic target of APS. Although Koike et al suggested that the phosphatidylserine-prothrombin complex (PS/PT) is one of the major target antigens of $\mathrm{LA}^{2}{ }^{2}$ the clinical significance of anti-PS/PT $\mathrm{Ab}$ is still controversial. ${ }^{4} \mathrm{~A}$ previous study reported that the prevalence of anti-CL/ $\beta_{2}$ GPI $\mathrm{Ab}$ was $10 \%$ and was significantly associated with isolated pulmonary hypertension in patients with SSc, ${ }^{5}$ but the clinical relevance of antiphospholipid Abs has not been established in SSc. In this study we investigated whether anti-PS/PT Ab can be detected in patients with SSc, and whether this Ab correlates with the clinical or serological features of the disease.

\section{METHODS}

\section{Serum samples}

Serum samples were obtained from 112 Japanese patients with SSc (99 women and 13 men, mean (SD) age 40 (19) years) who had attended Kanazawa University Hospital between 1995 and 2003. All patients fulfilled the criteria proposed by the American College of Rheumatology (ACR). ${ }^{6}$ Antitopoisomerase I Ab was positive for 40, anticentromere $\mathrm{Ab}$ for $4 \mathrm{l}$, anti-RNA polymerase I/III Ab for 10, and antiUIRNP Ab for 8. The mean (SD) disease duration of patients with SSc was $5.4(7.2)$ years. None of the patients with SSc had been treated with steroids, D-penicillamine, or immunosuppressive drugs when blood samples were taken at their first visit. Thirty patients with systemic lupus erythematosus (SLE; 27 women and 3 men; age 37 (14) years) who fulfilled the ACR criteria ${ }^{7}$ were also examined as disease controls in this study. In addition, 33 healthy Japanese people (29 women and 4 men; age 43 (10) years) served as normal controls.

\section{Clinical assessment}

Complete medical histories, physical examinations, and laboratory tests were conducted for all patients. Skin score was measured by the modified Rodnan total skin thickness score (mTSS). ${ }^{8}$ Organ system involvement was defined as described previously ${ }^{9}$ : lung = bibasilar pulmonary fibrosis on chest radiography and high resolution computed tomography; oesophagus = hypomotility shown by barium radiography; joint = inflammatory polyarthralgias or arthritis; heart = pericarditis, congestive heart failure, or arrhythmias requiring treatment; kidney $=$ malignant hypertension and rapidly progressive renal failure without any other explanation; and muscle = proximal muscle weakness and raised serum creatine kinase.

A pulmonary function test, including vital capacity (VC) and transfer factor for carbon monoxide (TLCO), was also carried out. When the TLCO and VC were $<75 \%$ and $<80 \%$, respectively, of the predicted normal values, they were considered to be abnormal. Isolated pulmonary hypertension was defined as clinical evidence of pulmonary hypertension and increased mean estimated right ventricular pressure (>35 mm Hg) documented by Doppler echocardiography in the absence of severe pulmonary fibrosis. The Kanazawa University Graduate School of Medical Science approved the protocol, and informed consent was obtained from all patients.

\section{ELISA for anti-PS/PT Abs}

Anti-PS/PT IgG Abs were measured by a specific enzyme linked immunosorbent assay (ELISA; Medical and Biological Laboratories, Nagoya, Japan), according to the manufacturer's protocol. Briefly, the serum samples diluted 1:101 were added to 96 well plates coated with PS/PT and

Abbreviations: $A b$, antibody; $A C R$, American College of Rheumatology; APS, antiphospholipid antibody syndrome; $\beta_{2}$ GPI, $\beta_{2}$-glycoprotein I; CL, cardiolipin; ELISA, enzyme linked immunosorbent assay; LA, lupus anticoagulant; mTSS, modified Rodnan total skin thickness score; PS, phosphatidylserine; PT, prothrombin; SLE, systemic lupus erythematosus; SSc, systemic sclerosis; TLCO, carbon monoxide transfer factor; VC, vital capacity 


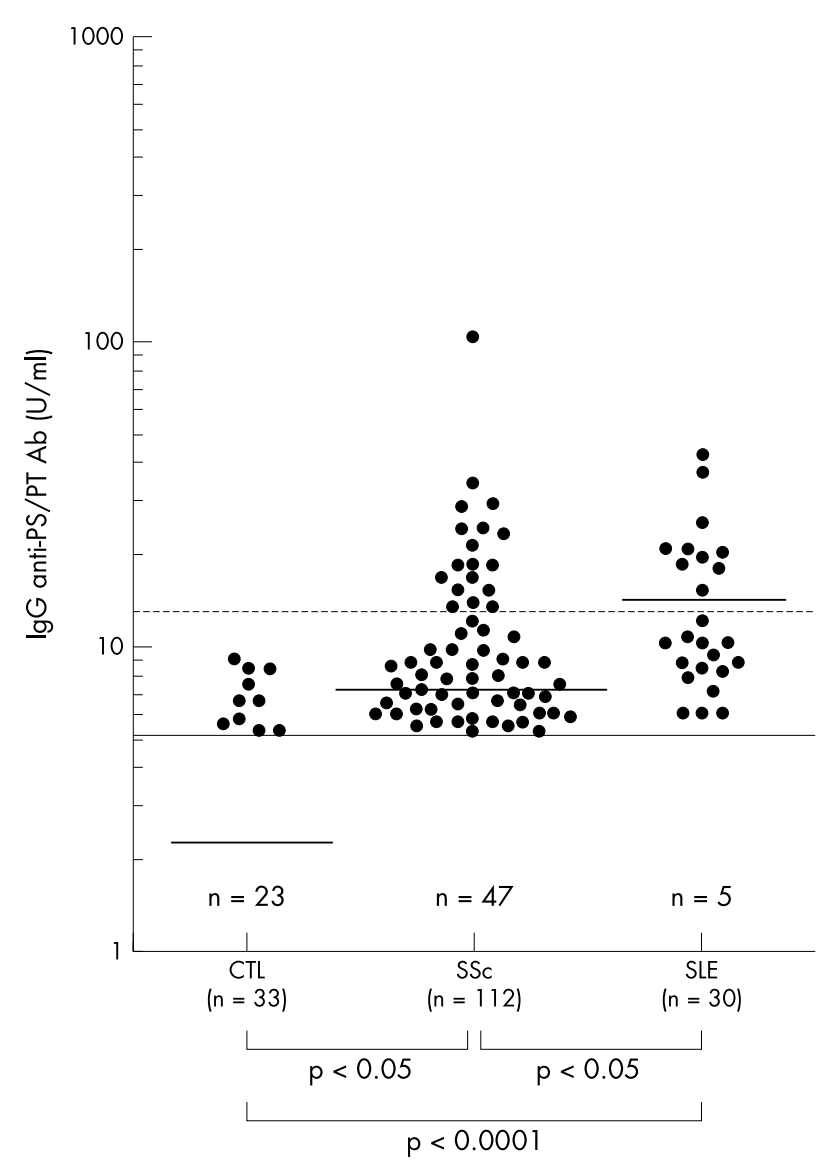

Figure 1 Abs against PS/PT in serum samples from patients with SSc, $\mathrm{SLE}$, and normal controls (CTL). Anti-PS/PT Ab levels were determined by ELISA. The horizontal line shows the detection limit $(5 \mathrm{U} / \mathrm{ml})$ and the number below the line indicates the number of patients with undetectable levels. The broken line represents the cut off value $(12 \mathrm{U} / \mathrm{ml})$. The short bar indicates the mean value in each group.

incubated for 1 hour at $20^{\circ} \mathrm{C}$. After washing, the bound Abs were detected with peroxidase conjugated antihuman IgG Ab. Colour was developed with 3,3',5,5'-tetramethylbenzidine and $\mathrm{H}_{2} \mathrm{O}_{2}$ and the plates were read at $450 \mathrm{~nm}$. The detection limit was $5 \mathrm{U} / \mathrm{ml}$. Anti-CL/ $\beta_{2}$ GPI Ab was measured using ELISA kits (Yamasa, Tokyo, Japan). LA was determined according to the guidelines recommended by the Subcommittee on Lupus Anticoagulant/Phospholipid Dependent Antibodies. ${ }^{10}$

\section{Statistical analysis}

Statistical analysis was performed with a Mann-Whitney U test for comparison of Ab levels, Fisher's exact probability test for comparison of frequencies, and Bonferroni test for multiple comparisons. A value of $p<0.05$ was considered significant. All data are shown as mean (SD).

\section{RESULTS}

Anti-PS/PT Ab levels in SSc

Patients with SSc exhibited mean IgG anti-PS/PT Ab levels that were $238 \%$ higher than those in normal controls (7.1 (11.8) $v 2.1$ (3.3) U/ml, p<0.05), but were $43 \%$ lower than those in patients with SLE (12.5 (10.4) U/ml, p<0.05; fig l). Values higher than the mean+3 SD $(12 \mathrm{U} / \mathrm{ml})$ of the normal control serum samples were considered positive in this study. IgG anti-PS/PT Ab was found in 18/112 (16\%) patients with SSc and 10/30 (33\%) patients with SLE, although anti-PS/PT
Table 1 Antiphospholipid Abs in patients with SSc

\begin{tabular}{|c|c|c|c|c|}
\hline $\begin{array}{l}\text { Patient } \\
\text { No }\end{array}$ & Thrombosis & $\begin{array}{l}\text { Anti-PS/PT Ab } \\
\text { (U/ml) }\end{array}$ & $\begin{array}{l}\text { Lupus } \\
\text { anticoagulant }\end{array}$ & $\begin{array}{l}\text { Anti-CL/ } \\
\text { B2GPI Ab }\end{array}$ \\
\hline 1 & + Deep vein & +104 & - & - \\
\hline 2 & + Deep vein & +35.2 & + & - \\
\hline 3 & - & +29.5 & + & - \\
\hline 4 & - & +29 & - & - \\
\hline 5 & + Heart & +24.5 & + & - \\
\hline 6 & - & +24.5 & ND & ND \\
\hline 7 & + Brain & +22 & + & - \\
\hline 8 & + Brain & +19.3 & + & + \\
\hline 9 & + Deep vein & +19.1 & - & - \\
\hline 10 & - & +18.2 & + & - \\
\hline 11 & - & +17 & - & - \\
\hline 12 & - & +17 & + & - \\
\hline 13 & - & +15.4 & ND & ND \\
\hline 14 & - & +15.1 & - & - \\
\hline 15 & - & +14 & - & - \\
\hline 16 & - & +13.3 & + & - \\
\hline 17 & - & +13.2 & + & - \\
\hline 18 & - & +13 & ND & ND \\
\hline 19 & - & -9.1 & + & + \\
\hline 20 & + Heart & -8.8 & + & - \\
\hline 21 & + Deep vein & -8.8 & + & + \\
\hline 22 & - & -8.1 & + & - \\
\hline 23 & - & -7.6 & + & + \\
\hline 24 & - & -6.8 & + & - \\
\hline 25 & + Deep vein & -6.2 & + & - \\
\hline 26 & - & $-<5$ & + & - \\
\hline 27 & - & $-<5$ & + & ND \\
\hline 28 & - & $-<5$ & + & - \\
\hline 29 & - & $-<5$ & + & - \\
\hline 30 & - & $-<5$ & + & - \\
\hline
\end{tabular}

IgG Ab was not detected in any normal controls. Thus, anti$\mathrm{PS} / \mathrm{PT}$ Ab was positive in some patients with SSc.

\section{Anti-PS/PT Ab in patients with SSc and APS}

Table 1 gives details of patients with SSc who were positive for either anti-PS/PT Ab, LA, or anti-CL/ $\beta_{2}$ GPI Ab. Of 112 patients with SSc who were assessed for anti-PS/PT Ab, LA and anti-CL/ $\beta_{2}$ GPI Ab were examined in 81 and 60 patients, respectively. LA and anti-CL/ $\beta_{2}$ GPI Ab were detected in $21 / 81$ $(26 \%)$ and $4 / 60(7 \%)$ patients with SSc, respectively. Of 18 patients with SSc with anti-PS/PT Abs, one patient had both $\mathrm{LA}$ and anti-CL $/ \beta_{2}$ GPI $\mathrm{Ab}$ and eight had LA alone. Six patients with anti-PS/PT Abs had neither LA nor anti-CL/ $\beta_{2}$ GPI Ab. Unexpectedly, the titre of anti-PS/PT Ab in patients with SSc with LA $(\mathrm{n}=21)$ was not significantly different from those without LA $(\mathrm{n}=60,8.4$ (9.3) $v 6.8(14.0) \mathrm{U} / \mathrm{ml})$.

The 30 patients with SSc positive for anti-PS/PT Ab or LA or anti-CL/ $\beta_{2}$ GPI Ab were screened for clinical signs of APS by magnetic resonance imaging, ventilation/perfusion pulmonary scintigraphy, electrocardiography, echocardiography, and phlebography. None of the 30 patients had a history of intrauterine fetal loss. Thromboembolism was detected in $9 / 30(30 \%)$ patients with antiphospholipid Abs. Although five patients (cases 1, 5, 20, 21, and 25) had clinical symptoms of thromboembolism, the other four patients were asymptomatic. Thromboembolism was detected in 6/18 (33\%) patients with anti-PS/PT Ab with or without LA and anti$\mathrm{CL} / \beta_{2}$ GPI Ab. These six patients, in particular, had anti-PS/PT Abs that were higher than $19 \mathrm{U} / \mathrm{ml}$. Although 2/82 (2\%) patients without antiphospholipid $\mathrm{Ab}$ had symptomatic thromboembolism, other asymptomatic patients were not screened for thromboembolism. Importantly, two patients (cases 1 and 9) with deep vein thrombosis had only anti-PS/ PT Ab but not LA or anti-CL/ $\beta_{2}$ GPI Ab. Therefore, anti-PS/PT $\mathrm{Ab}$ may need to be examined for the careful detection of APS in patients with SSc. 
Table 2 Clinical data of patients with SSc showing positive anti-PS/PT Ab

\begin{tabular}{lll} 
& $\begin{array}{l}\text { Anti-PS/ } \\
\text { PT Ab (+) } \\
\text { (n= 18) }\end{array}$ & $\begin{array}{l}\text { Anti-PS/ } \\
\text { PT Ab (-) } \\
\text { (n=94) }\end{array}$ \\
Clinical data & $41(20)$ & $46(15)$ \\
\hline Age at onset (years), mean (SD) & $4: 14$ & $9: 85$ \\
Sex (male:female) & $5.4(5.6)$ & $5.3(7.0)$ \\
Duration (years), mean (SD) & & \\
Skin & $14.7(12.0)$ & $10.7(9.3)$ \\
mTSS & $72^{*}$ & 32 \\
Pitting scars & $39^{*}$ & 12 \\
Digital skin ulcer & & \\
Lung & $78^{*}$ & 36 \\
Pulmonary fibrosis & $56^{*}$ & 18 \\
Pulmonary hypertension & $61^{*}$ & 24 \\
Decreased \%VC & $89^{*}$ & 54 \\
Decreased \%TLCO & 89 & 63 \\
Oesophagus & 39 & 20 \\
Heart & 17 & 3 \\
Kidney & 44 & 22 \\
Joint & 11 & 14 \\
Muscle & & 32 \\
Laboratory findings & 56 & 39 \\
Antitopoisomerase I Ab & 22 & 9 \\
Anticentromere Ab & 11 & 12 \\
Anti-RNA polymerase Ab & 6 & \\
Anti-U1RNP Ab & 33 & \\
Raised ESR & 22 & \\
Raised CRP & & \\
\hline
\end{tabular}

Values are percentages unless otherwise stated. mTSS, modified Rodnan total skin thickness score; ESR, erythrocyte sedimentation rate; CRP, C reactive protein.

${ }^{*} \mathrm{p}<0.01 v$ patients with $\mathrm{SSc}$ with negative anti-PS/PT Ab.

\section{Association of anti-PS/PT Ab with clinical features}

The prevalence of patients with SSc with positive IgG anti-PS/ PT Ab was significantly higher in patients with SSc with the clinical signs of peripheral ischaemia, including pitting scars and digital skin ulcer than in patients without it (table 2). Furthermore, a greater proportion of patients with SSc and positive IgG anti-PS/PT Ab had lung disease, including pulmonary fibrosis and isolated pulmonary hypertension, than patients without the Ab. Consistently, decreased \%VC and \%TLCo were more commonly found in patients with anti$\mathrm{PS} / \mathrm{PT} \mathrm{Ab}$ than in patients without the Ab. However, anti-PS/ PT Ab levels were not significantly associated with mTSS, a semiquantitative measure of skin sclerosis. Thus, anti-PS/PT $\mathrm{Ab}$ was associated with peripheral ischaemia and lung disease but not with the extent of skin sclerosis in patients with SSc.

\section{DISCUSSION}

In this study IgG anti-PS/PT autoAb was detected in 16\% of patients with SSc (fig 1). Thirty three per cent of patients with SSC and the IgG anti-PS/PT Ab had episodes of thromboembolism with or without LA and anti-CL $/ \beta_{2}$ GPI $\mathrm{Ab}$ (table 1). The mechanism by which anti-PS/PT Abs enhance thromboembolism remains unclear. It has been speculated that anti-PT Abs inhibit thrombin mediated prostacyclin, that PT binds to anionic phospholipids exposed on the endothelial cells, and that anti-PT Abs activate endothelial cells and induce procoagulant substances through PT. ${ }^{11}$ Recent studies have demonstrated that antiPS/PT Abs show LA activity and are closely associated with LA. $^{2}{ }^{12}$ However, anti-PS/PT Ab was not significantly associated with LA in this study. This discrepancy may be due to differences and heterogeneity of the detection methods or patients. Alternatively, this may reflect the fact that antiPS/PT Abs are also a heterogeneous group of Abs that can be divided into Abs with and without LA activity, as demonstrated in anti-PT Ab. ${ }^{13}$ Two of six patients with SSc with IgG anti-PS/PT Ab had thromboembolism, although these two patients had neither LA nor anti-CL/ $\beta_{2}$ GPI $A b$ (table 1). Therefore, it may be useful to include the evaluation of anti-PS/PT Ab in the screening of APS, especially in patients negative for both LA and anti-CL/ $\beta_{2}$ GPI Ab.

The results of this study suggest that patients with SSC who have IgG anti-PS/PT Abs have unique clinical features, and may represent a group of patients with SSc with peripheral ischaemia and lung disease (table 2). In contrast, anti-PS/PT Ab was not associated with the severity of skin sclerosis. In a large study of the European Phospholipid Project Group, several clinical manifestations had a prevalence of $1-6 \%$ in patients with APS. ${ }^{14}$ Interestingly, these manifestations included pulmonary hypertension, fibrosing alveolitis, and ischaemic cutaneous lesions, such as skin ulcer and digital gangrene. Whether anti-PS/PT Abs have some role in these clinical symptoms of SSc or only reflect more basic underlying immunological abnormalities is unknown. Recent studies suggested that antiphospholipid Abs induce thromboembolism by activating endothelial cells. ${ }^{15}$ Activated endothelial cells express tissue factor, plasminogen activator inhibitor-1, adhesion molecules, and proinflammatory cytokines, leading to procoagulant state and inflammation. ${ }^{15}$ Therefore, our findings may reflect additional pathogenic roles of anti-PS/PT Ab in the peripheral ischaemia and pulmonary involvement of SSc, by activating endothelial cells, leading to a subsequent procoagulant state and inflammation. The assessment of anti-PS/PT Ab in addition to $\mathrm{LA}$ and anti-CL/ $\beta_{2}$ GPI $\mathrm{Ab}$ may contribute to a better recognition of APS and clinical features in SSc.

\section{ACKNOWLEDGEMENTS}

We thank Ms M Matsubara for technical assistance.

\section{Authors' affiliations}

M Hasegawa, S Sato, K Yanaba, K Komura, K Takehara, Department of Dermatology, Kanazawa University Graduate School of Medical Science, Kanazawa, Japan

M Yamazaki, Department of Internal Medicine (III), Kanazawa University Graduate School of Medical Science, Kanazawa, Japan

Correspondence to: Dr M Hasegawa, Department of Dermatology, Kanazawa University Graduate School of Medical Science, 13-1 Takaramachi, Kanazawa, Ishikawa 920-8641, Japan; minoruha@ derma.m.kanazawa-u.ac.jp

Accepted 27 January 2004

\section{REFERENCES}

1 Harris EN, Chan JK, Asherson RA, Aber VR, Gharavi AE, Hughes GR. Thrombosis, recurrent fetal loss, and thrombocytopenia. Predictive value of the anticardiolipin antibody test. Arch Intern Med $1986 ; 146: 2153-6$

2 Atsumi T, leko M, Bertolaccini ML, Ichikawa K, Tsutsumi A, Matsuura E, et al. Association of autoantibodies against the phosphatidylserineprothrombin complex with manifestations of the antiphospholipid syndrome and with the presence of lupus anticoagulant. Arthritis Rheum 2000;43: 1982-93.

3 Amengual O, Atsumi T, Koike T. Specificities, properties, and clinical significance of antiprothrombin antibodies. Arthritis Rheum 2003;48:886-95.

4 Galli M, Luciani D, Bertolini G, Barbui T. Anti-beta 2-glycoprotein I, antiprothrombin antibodies, and the risk of thrombosis in the antiphospholipid syndrome. Blood 2003;102:2717-23.

5 Ihn H, Sato S, Fujimoto M, Kikuchi K, Igarashi A, Soma Y, et al. Measurement of anticardiolipin antibodies by ELISA using $\beta 2$-glycoprotein I ( $\beta 2-\mathrm{GPI})$ in systemic sclerosis. Clin Exp Immunol 1996;105:475-9.

6 Subcommittee for scleroderma Criteria of the American Rheumatism Association Diagnostic and Therapeutic Criteria Committee. Preliminary criteria for the classification of systemic sclerosis (scleroderma). Arthritis Rheum 1980;23:581-90.

7 Tan EM, Cohen AS, Fries JF, Masi AT, McShane DJ, Rothfield NF, et al. The 1982 revised criteria for the classification of systemic lupus erythematosus. Arthritis Rheum 1982;25:1271-7. 
8 Clements PJ, Lachenbruch PA, Seibold JR, Zee B, Steen VD, Brennan P, et al Skin thickness score in systemic sclerosis: an assessment of interobeservar variability in 3 independent studies. J Rheumatol 1993;20:1892-6.

9 Sato S, Ihn H, Kikuchi K, Takehara K. Antihistone antibodies in systemic sclerosis: association with pulmonary fibrosis. Arthritis Rheum 1994;37:391-4.

10 Brandt JT, Triplett DA, Alving B, Scharrer I. Criteria for the diagnosis of lupus anticoagulants: an update. On behalf of the Subcommittee on Lupus Anticoagulant/Antiphospholipid Antibody of the Scientific and Standardisation Committee of the ISTH. Thromb Haemost 1995;74:1 185-90.

11 Arnout J. The pathogenesis of the antiphospholipid syndrome: a hypothesis based on parallelisms with heparin-induced thrombocytopenia. Thromb Haemost 1996;75:536-41.
12 Fleck RA, Rapaport SI, Rao LV. Anti-prothrombin antibodies and the lupus anticoagulant. Blood 1988;72:512-19.

13 Horbach DA, van Oort E, Derksen RH, de Groot PG. The contribution of antiprothrombin-antibodies to lupus anticoagulant activity-discrimination between functional and non-functional anti-prothrombin-antibodies. Thromb Haemost 1998;79:790-5.

14 Cervera R, Piette JC, Font J, Khamashta MA, Shoenfeld Y, Camps MT, et al. Antiphospholipid syndrome: clinical and immunologic manifestations and patterns of disease expression in a cohort of 1,000 patients. Arthritis Rheum 2002;46:1019-27.

15 Meroni PL, Raschi E, Testoni C. Endothelium as a target for antiphospholipid antibodies and for therapeutical intervention. Autoimmun Rev 2002;1:55-60. 